\title{
Seismic Analysis of Civil Structures
}

\author{
Xiao Songtao \\ Yunnan Technology and Business College, Yunnan, China, 651701
}

Keywords: High-rise building, seismic performance, improved design

\begin{abstract}
The country is located in a country with multiple seismic zones. It is adjacent to the Pacific seismic belt in the east and the Eurasian seismic belt in the south. The earthquake is widely distributed. The frequency of earthquake activity is high and the magnitude is large. It is a country with severe earthquake disasters in the world. One. Therefore, with the development of the economy and the diversity of social needs, the height of the building is getting higher and higher, the body shape becomes more complicated and the seismic design becomes more and more difficult. How to improve the level of seismic design of building structures from the actual situation of earthquake environment and social development in China, making it safer, more reliable, more reasonable and economical, is an important task for many engineers. This paper combines the author's many years of architectural design experience to explain the importance of improving the seismic resistance of high-rise buildings in China and gives some design ideas and improvement measures.
\end{abstract}

\section{Introduction}

The seismic magnitude of the building structure is divided according to the intensity of the earthquake. In China, the earthquake is divided into six levels: 3 is a small earthquake, 3 to 4.5 are sensitive earthquakes and 4.5 to 6 are moderately strong earthquakes, $6 \sim 7$ is a strong earthquake, 7-8 is a large earthquake and 8 or more is a huge earthquake. It is an experience of the country based on relevant historical, geographical and geological experience data, after exploration and verification. Numerical, it is a geographical concept. The anti-seismic fortification has buildings of Class A, B and D. The earthquake resistance level of most houses in China is 8 degrees, which can resist the impact of the magnitude 6 earthquake. Strong earthquakes, especially large earthquakes, are small-probability events that are highly destructive but have little chance of occurrence. In China alone, most parts of China are threatened by devastating earthquakes. Since the founding of New China, the Tangshan earthquake of 7.8 in 1976 and the Wenchuan earthquake of magnitude 8 in 2008 have caused great losses to people's lives and property. At the same time, however, it should also be noted that most of the buildings in the seismic fortification zone were not attacked by major earthquakes throughout their service. To sum up: Earthquakes are random vibrations with complexities and uncertainties that are difficult to grasp. It is difficult to accurately predict the characteristics and parameters of earthquakes encountered by buildings. However, as a product of social production and the material life of human beings, high-rise buildings are the inevitable result of industrialization, commercialization and urbanization of modern society. This requires the structural engineer to perform structural analysis and design according to the seismic design requirements, in order to achieve the best in terms of strength, stiffness, ductility and energy consumption, so as to achieve economically "small earthquakes are not bad, medium earthquakes" The design concept of repairable, big shock does not fall ${ }^{[1]}$.

\section{Select foundation and site}

When selecting the foundation, in general, rock, semi-rock and dense foundation soil are the most favorable for earthquake resistance of buildings and it is the best construction site; and the bases that are unfavorable to earthquake resistance of buildings are: bases with thick and weak alluvial soil sites. The base where the soil liquefaction leads to uneven settlement of the foundation, 
the base with the natural vibration period and the basic period of the building structure; when selecting the construction site, the relevant information of seismic activity, engineering geology and seismic geology should be mastered according to the project needs and the earthquake-resistant advantage Comprehensive evaluation of unfavorable and dangerous locations. For unfavorable areas, avoidance requirements should be made; when it is impossible to avoid, effective measures should be taken to reduce hidden dangers and eliminate disadvantages ${ }^{[2]}$.

\section{Improve the earthquake resistance of the building itself}

The structural design of the building is a key factor influencing the seismic effect. If the building is to achieve seismic resistance, proper structural design must be carried out to ensure reasonable seismic measures. This article only discusses the following three aspects:

1) Pay attention to regularity. Structural engineers always hope that the structural rules, symmetry and force transmission path designed are direct and simple. However, with the development of the economy and the diversity of social needs, the diversification requirements of the owners and architects for the use of functions and the flat and façade of the building inevitably lead to higher building heights and more complex shapes. Engineers have to adopt The more complex high-rise building structure makes the seismic design of high-rise buildings more difficult. However, the layout of the building and its anti-lateral force structure should be as simple and regular as possible and the stiffness and bearing capacity should be evenly distributed.

In the shape, the shape is convex and concave, the center of mass and the center of the structure are not heavy and the corner stress is concentrated during the earthquake and the torsional vibration is obvious, resulting in a member with less rigidity from the center of the core and the amount of lateral shift is increased and the level of sharing is balanced. Seismic forces and significant increases are prone to damage and even cause the entire structure to collapse due to structural failure of one side ${ }^{[3]}$.

2) Choose building materials. From the perspective of seismic design, the seismic level of the structural system is essentially to control the ductility requirements of different structures on a macroscopic basis. For example, ordinary steel bars should be selected from steel bars with good ductility, toughness and weldability; strength grades of ordinary steel bars, longitudinal HRB400 and HRB335 hot-rolled steel bars should be used for the stressed steel bars. HRB335, HRB400 and HPB235 hot-rolled steel bars should be used for the hoops. From the degree of earthquake damage: the magnitude of the effect of the earthquake on the structure is almost proportional to the mass of the structure. Generally speaking, under the same conditions, the mass is large, the earthquake effect is large, the degree of earthquake damage is large, the mass is small, the earthquake effect is small and the earthquake damage is small. Therefore, lightweight materials such as porous bricks, silicate blocks, ceramsite concrete, aerated concrete slabs, hollow plastic sheets, etc. are widely used in the slabs, walls, frames, partitions, retaining walls and roofing members of buildings. Will significantly improve the seismic performance of buildings ${ }^{[4]}$.

3) About isolation and energy dissipation. Seismic isolation is to isolate an earthquake, that is, to provide a layer of isolation between the foundation of the building and the superstructure to isolate the house from the foundation and to isolate the transmission of ground motion energy to the building to reduce the seismic response of the building structure. When the earthquake is realized, only minor movements and deformations occur in the building to ensure the safety of the building. Energy dissipation is achieved by providing energy dissipating components in the building, so that part of the energy input into the building by the earthquake is consumed by the energy dissipating components and part of the energy is borne by the kinetic energy and deformation energy of the structure, thereby achieving the purpose of reducing structural seismic response. With the continuous improvement of the seismic requirements of high-rise buildings, the application of "ductile structural systems" has become increasingly limited and traditional seismic structural systems and theories are increasingly difficult to meet the requirements. Because the isolation and energy dissipation and various damping control systems have the superiority that traditional seismic systems are incomparable, they will be more and more widely used in future building structures. 
Dampers have emerged in the design of isolation and vibration isolation. Through the use of dampers, shock absorption and energy absorption, the damage of high-rise buildings can be avoided or weakened. Vibration isolation structure shown in Figure 1.

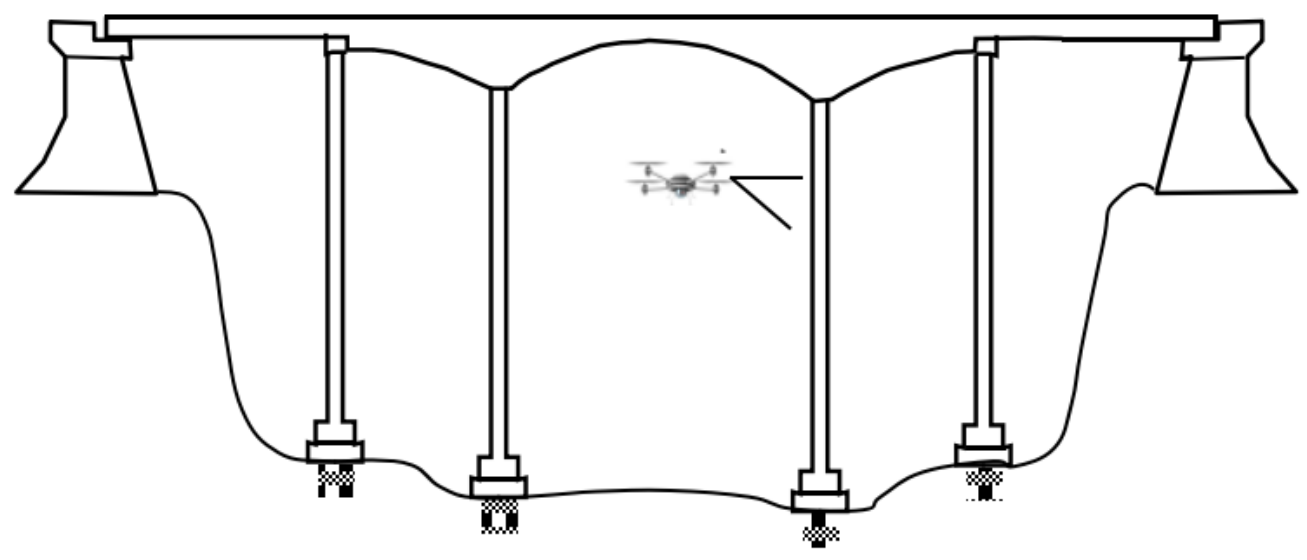

Figure 1: Vibration isolation structure

\section{Commonly used auxiliary structure design}

In order to face the sudden earthquake attack, high-rise buildings also need other auxiliary structure design, part of the structure is shown in Figure 2.
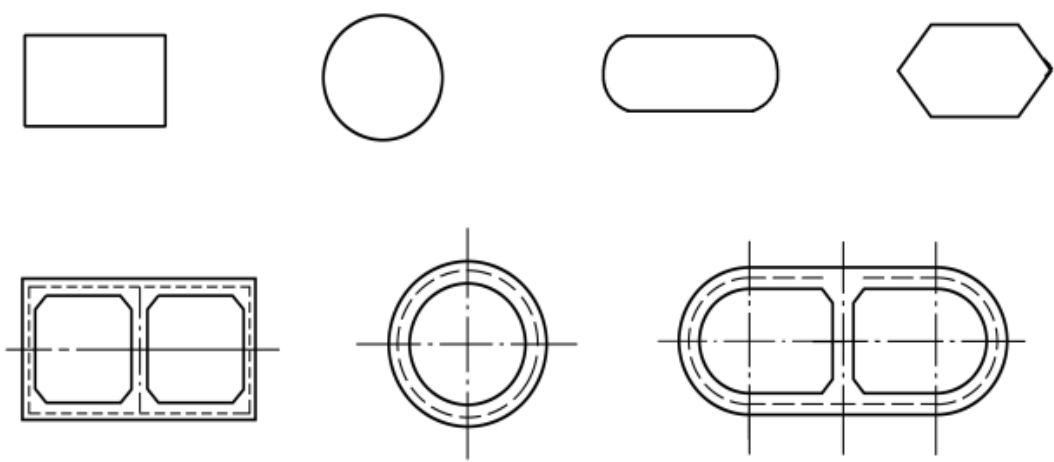

Figure 2: Part of the structure

1) Set up multiple anti-seismic defense lines. After each strong earthquake, there are often multiple aftershocks. Therefore, if there is only one fortification in the seismic design process of the building, after the first line of defense is destroyed, there is no backup second, third or even more defense lines. By blocking the force of the earthquake, it is difficult to avoid the collapse of high-rise buildings.

2) Deal with the strong and weak relationship of structural members. Consciously establish a series of distributed yield zones. The main energy-consuming components should have high ductility and appropriate stiffness so that the structure can absorb and consume a large amount of seismic energy, such as:

After the main energy-consuming components are yielded in the same floor, the other lateral-resisting members are still in the elastic stage, so that the "effective yielding" is maintained for a longer period, ensuring the ductility and collapse resistance of the structure ${ }^{[5]}$.

3) Adopt "frame shear wall". The frame shear wall has a multi-channel anti-seismic structure with better performance and the shear wall is also the main anti-side force member of the first anti-seismic line. Therefore, the shear wall should be sufficient to ensure that it has a high bearing capacity, not less than $50 \%$ of the seismic overturning moment at the bottom of the tall building. At the same time, in order to withstand the seismic action of redistribution after shear wall cracking, the seismic shear force of any layer frame part according to the joint work of frame and wall should 
not be less than $20 \%$ of the total seismic shear force at the bottom of the structure and the seismic shear of each layer of the frame. A smaller value of 1.5 times the maximum value of the force.

4) Improve the shock resistance of the short column. High-rise buildings require seismic fortification in addition to strength and stiffness requirements, as well as ductility requirements. Improving the seismic performance of concrete short columns is mainly to improve the ductility of concrete short columns. Here are a few aspects: the use of high-strength concrete to increase the compressive bearing capacity of the column, reduce its axial compression ratio; the concrete is filled into the thin-walled circular steel pipe to form the concrete-filled steel tube, so that the concrete is in a three-way compression state, so that the concrete resistance The compressive strength and ultimate compressive strain are greatly improved and the ductility is significantly improved. Because the flexural capacity of the short column is much larger than the shear bearing capacity, it is often broken due to shearing under earthquake action and its bending resistance Strength cannot be fully utilized. Therefore, the split column method can be used to weaken the bending strength of the short column so that the bending strength corresponds to or slightly lower than the shear strength. In this way, under the action of the earthquake, the column will first reach the bending strength, thus exhibiting a ductile state of destruction.

\section{Conclusion}

Since the 20th century, China's building structure has made great achievements in earthquake resistance. To be further improved and developed on the basis of existing ones, it is necessary to use not only traditional technologies but also new technologies. For example, computer simulation of seismic test, the fabricated model or structural components are placed on the simulated seismic vibration table and the platform inputs a certain deterministic seismic record, which can simulate the real earthquake resistance effect, help scientifically improve various factors and effectively resist earthquakes. In addition to improving the design method, we also need to constantly learn new design concepts to achieve the goal of keeping pace with the times.

\section{References}

[1] P. Güneş,A.E. Aksu,J. Hall. Structural framework and deformation history of the western Cyprus Arc[J]. Tectonophysics,2018,744.

[2] Fei-fei Yan,Qi Zhu,Qian-Li Li,Ru-Fen Zhang,Chun-Lin Ma. Triorganotin coordination polymers based on three dicarboxylate ligands containing flexible $\mathrm{S}<$ ce:glyph name="sbnd" $>\mathrm{S}$ bonds: synthesis, structures and in vitro anti-tumor activity[J]. Journal of Organometallic Chemistry,2019,880.</ce:glyph>

[3] Yun-Shan Xue,Jia-Xin Yang,Ju-Qing Han,Su-Su Liu,Ying-Ying Cui,Yu Tan,Yu-Peng Hua,Wen-Min Wang. Solvent-induced two Dy 2 compounds with different<ce:hsp $\mathrm{sp}=$ "0.25">structures showing distinct slow magnetization relaxation behaviors[J]. Polyhedron,2018.</ce:hsp>

[4] Geumhee Yun,Hansaem Lee, Yongsuk Hong,Sungpyo Kim,Glen T.Daigger,Zuwhan Yun.The difference of morphological characteristics and population structure in PAO and DPAO granular sludges[J].Journal of Environmental Sciences,2019,76(02):388-402.

[5] Juan Cheng, Jin Lv,Hai Shun Wu. Structural, Electronic and Tunable Magnetic Properties of Transition Metal Doped Rh 8 Cluster from First Principles Calculation[J]. Journal of Cluster Science,2018,29(5). 